\title{
A New Optical Method for Characterizing Single Molecule Interactions based on Dark Field Microscopy
}

\author{
Heidelinde R. C. Dietrich, Bart J. Vermolen, Bernd Rieger, Ian T. Young and Yuval Garini \\ Quantitative Imaging Group, Department of Imaging Science \& Technology, Faculty of Applied Sciences, \\ Delft University of Technology, Lorentzweg 1, NL-2628 CJ Delft, The Netherlands
}

\begin{abstract}
Single-molecule techniques continue to gain in popularity in research disciplines such as the study of intermolecular interactions. These techniques provide information that otherwise would be lost by using bulk measurements that deal with a large number of molecules. We describe in this report the motion of tethered DNA molecules that have been tagged with gold nanobeads and observed under dark field microscopy to study single molecular interactions (SMI). We further report on the derivation and use of several physical parameters and how these parameters change under differing experimental conditions.
\end{abstract}

Keywords: single molecule detection, gold nanobeads, molecular interactions, dark field microscopy, tethered-particle motion

\section{INTRODUCTION}

In recent years, single molecule techniques have increased in popularity especially in fundamental biochemical and biophysical research. These techniques allow the characterization of events that are otherwise hidden if ensembleaveraged methods are applied. The static and dynamic heterogeneity of a population/system can be examined. Single molecule studies deliver distributions rather than average values. They give information on transition states, time trajectories, conformational changes, etc. These techniques require small sample sizes compared to conventional biochemical assays ${ }^{1}$.

DNA-protein interactions are of great interest due to their importance in cellular processes. They control events such as DNA replication, DNA repair and transcription regulation. Research using single molecule interactions not only has fundamental importance but also relevance in applied research, for example, the development of effective therapies for various types of cancer.

The study of DNA-protein interactions includes the screening for DNA-binding protein, the analysis of reaction kinetics, and the determination of the influence of changing environmental conditions on the DNA binding event ${ }^{2-4}$.

There are a number of bulk biochemical and classical biophysical methods that allow the study of these interactions, such as electrophoretic mobility shift assays (EMSA), DNA footprinting and UV-VIS Spectroscopy. In the past twenty years, new techniques have been developed that allow the study of single molecules or single molecular events resulting in information on molecular mechanisms ${ }^{5,6}$. Optical and magnetic tweezers, for example, cannot only be used for the detection of single molecules but also for manipulating them and for the determination of associated forces. Both methods have been extensively used in the characterization of motor proteins, like kinesin and myosin ${ }^{6}$. There are single molecule methods that are based on fluorescence such as fluorescence resonance energy transfer (FRET) and fluorescence correlation spectroscopy (FCS). Other single molecule methods are based on physical phenomena such as surface plasmon resonance (SPR).

All of those techniques have their benefits and drawbacks. It is up to the researcher to choose the most suitable method for the detection and characterization of single molecule interactions.

\section{METHOD}

We have developed an optical method to study the dynamic behaviors associated with DNA-protein interactions. Our technique is based on tethered particle motion (TPM). One end of the DNA fragment is immobilized onto a gold slide

Ultrasensitive and Single-Molecule Detection Technologies II,

edited by Jorg Enderlein, Zygmunt K. Gryczynski, Proc. of SPIE Vol. 6444

644403, (2007) - 1605-7422/07/\$18 - doi: 10.1117/12.699040

Proc. of SPIE Vol. 6444 644403-1 
though disulfide chemistry. The other end is attached to a gold bead through biotin/anti-biotin interactions ${ }^{7,8}$. These sub-micron gold beads scatter polychromatic light when illuminated with white light.

In solution, the position of the bead is limited to a volume that depends on the length of the DNA molecule. The exact determination of this "action" volume results in information on the effective length of the DNA fragment and its possible variations due to the interaction with proteins or due to changes in environmental conditions such as the $\mathrm{pH}$ and salt concentration. Figure 1 illustrates how the volume might be effected by two different proteins, lacR and recA.

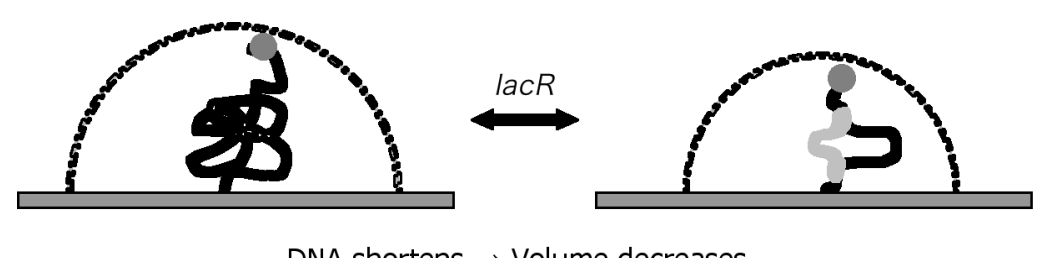

DNA shortens $\rightarrow$ Volume decreases

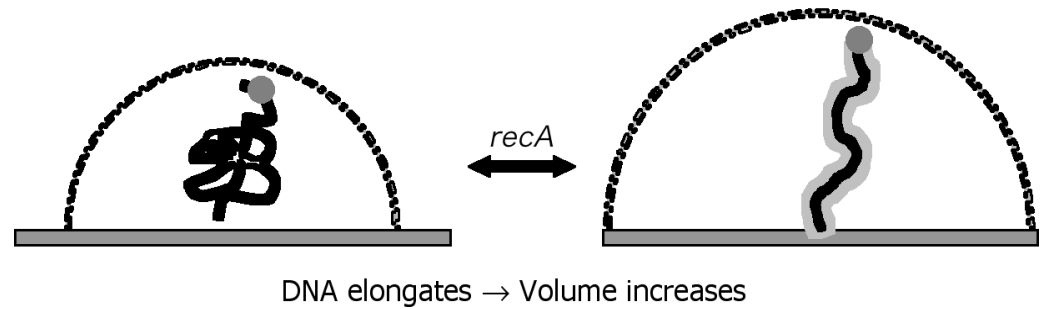

Figure 1. The Lactose repressor protein binds to the operator sequence on a dsDNA. It induces a loop, which decreases the length of the fragment, thus decreasing the action volume. The recA protein forms a filament around the dsDNA, resulting in an extended and stiffer DNA molecule.

The kinetics of E. coli RNA polymerase were first studied using TPM by Schafer et al. (1991). Finzi et al. (1995) measured DNA looping by the lactose repressor protein of E.coli in single fragments of double-stranded DNA. Many other research articles have been published on the use of TPM and the study of DNA-protein interactions. Most of these publications are based on video-microscopy in order to study biomolecular interactions. Others have focused mainly on the analysis of the data that have been acquired with the TPM method ${ }^{9-15}$.

In TPM, large polystyrene beads are usually used. The diameter of these beads are typically between a few hundred nanometers and $1 \mu \mathrm{m}$. Our method makes use of gold nanobeads $(\varnothing \approx 80 \mathrm{~nm})$. The metallic beads that we use (RLS beads, Invitrogen, The Netherlands) differentially scatter polychromatic light depending on the bead size. Metallic beads are well-suited for long term experiments as they neither bleach nor blink. They are commonly tagged with antibiotin antibodies.

The DNA fragments $(\approx 5 \mathrm{~kb})$ were amplified from a lambda DNA template using the Expand Long Template PCR System (Roche) and were modified with a disulfide group and a biotin molecule.

We use a darkfield microscope (Olympus BX51) with a 50x/0.8 air objective in order to detect the scattered light of the gold bead. The motion of the beads is detected with a 12 bit CCD camera (Hamamatsu, Orca-ER C4742-95). The CCD array consists of $1344 \times 1024$ pixels. Each pixel has a size of $6.45 \mu \mathrm{m} \times 6.45 \mu \mathrm{m}$. The CCD camera is controlled by SimplePCI from Compix Inc. Imaging Systems. The sampling density in $x$ - and $y$ - directions are: $\Delta_{\mathrm{x}}=0.13 \mu \mathrm{m}(7.7$ samples per $\mu \mathrm{m})$ and $\Delta_{\mathrm{y}}=0.13 \mu \mathrm{m}(7.7$ samples per $\mu \mathrm{m})$. The collected images are analyzed by MATLAB using our inhouse software package DIP Image ${ }^{16}$.

\section{EXPERIMENTS AND RESULTS}

Our results are based upon the detection of the constrained Brownian motion in solution of a tethered dsDNA fragment labeled with a gold nanobead. From the measured $x$ - and $y$-coordinates of the light scattered from a bead (Figure 2), 
various parameters can be derived such as the excursion of the DNA/bead system, the speed, and the diffusion characteristics. The ability to make accurate and precise measurements of these types of data has been extensively studied. In a recent publication, Ram et al. have shown, for example, that ten nanometer scale resolution is achievable ${ }^{17}$. Our experimental results agree with this prediction.

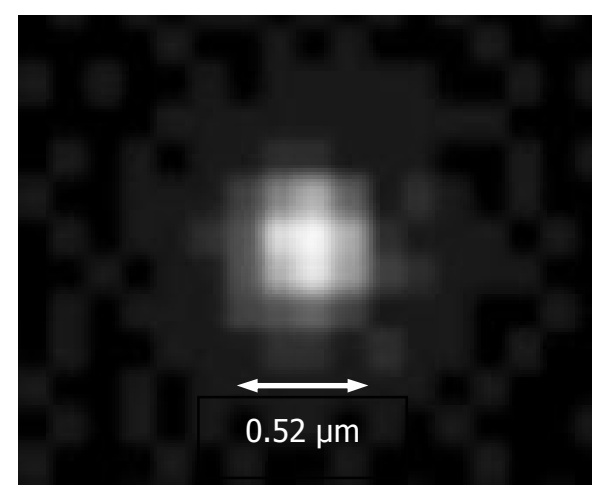

Figure 2. Image of light scattered from a gold bead whose diameter is $80 \mathrm{~nm}$. The width of the bead image is about 4 pixels $=0.52 \mu \mathrm{m}$.

All experiments were performed at ambient temperature. The dsDNA fragments used were 4861 base pairs long. A Tris/EDTA $(10 \mathrm{mM} / 1 \mathrm{mM})$ buffer with $\mathrm{pH} 8.0$ at $20{ }^{\circ} \mathrm{C}$ was used. For salt experiments, $\mathrm{MgCl}_{2}$ was dissolved in Tris$\mathrm{HCl}$ buffer $(10 \mathrm{mM}), \mathrm{pH} 8.0$ at $20^{\circ} \mathrm{C}$. Most experiments were carried out at a camera frame rate of $\approx 16$ frames per second depending on the size of the region of interest. (Larger regions-of-interest require a slightly lower frame rate.) The motion of the beads was monitored for 1500 to 2000 frames which was sufficient to achieve an accurate estimate of the dynamics of the movement of the tethered molecule.

TPM measurements rely on a bead that is usually rather large (up to $1 \mu \mathrm{m}$ ). This bead functions as a beacon for the molecular motion of the tethered biopolymer (DNA, RNA) ${ }^{18}$. The statistical properties of the macromolecule can, in principle, be altered by the reporter beacon depending upon its size. This is due to a volume-exclusion effect that arises from close proximity of the bead to the anchoring surface of the biopolymer. It is therefore of great importance to know if the size of the beacon influences a particular TPM measurement. Segall et al. have studied this problem and reported on the impact of the bead's size on the molecular movement and the derived parameters. They concluded that the excursion of the bead depends on a dimensionless quantity called the "excursion number"

$$
N_{R} \equiv R / \sqrt{L \xi / 3}
$$

where $R$ is the bead radius, $L$ is the contour length of the macromolecule and $\xi$ is the persistence length. The excursion number describes the bead's scaling behavior, giving information on whether molecule-dominated motion $\left(N_{R}<1\right)$ or bead-dominated motion $\left(N_{R}>1\right)$ predominates.

If we apply this concept to our system - with a contour length of $1653 \mathrm{~nm}(4861 \mathrm{bp})$, a persistence length of $50 \mathrm{~nm}$, and a bead radius of $40 \mathrm{~nm}$ - an excursion number of $N_{R}=0.24$ results. This implies that in all of our experiments the motion is molecule-dominated. Further, under the circumstances of our system, the fragment length could be shortened to $97 \mathrm{~nm}(285 \mathrm{bp})$ and we would still retain a molecule-dominated motion.

The root mean square radius, $r_{r m s}=\sqrt{\left\langle r^{2}\right\rangle}$, for a dsDNA tethered bead and for a stationary bead are shown in Table 1. The values are derived from the determination of the center-of-mass of the bead images in one data set. 
Table 1. rms radius values for a tethered bead and a stationary bead.

\begin{tabular}{|c|c|c|}
\hline & Tethered bead $[\mathbf{n m}]$ & Stationary bead [nm] \\
\hline$r_{r m s}=\sqrt{<r^{2}>}$ & 317 & 14 \\
\hline
\end{tabular}

The $r_{r m s}$ of a stationary bead is about 22 times smaller than a moving one. The values for an immobile bead can be seen as control values. They represent the error of the method/system due to stochastic sources such as the dark noise, electronic camera noise, quantization noise, and mechanical vibrations.

In Figure 3a,b the radial positions and the distribution of a moving and stationary bead are shown. In both cases, the excursion of the bead over 2000 frames shows no favored direction. The average radial distance, $\langle r\rangle$, of a moving, tethered bead is $286 \mathrm{~nm}$. An immobile bead, on the other hand, has a characteristic average radial distance of $13 \mathrm{~nm}$. This value can again be taken as a control value for noise.
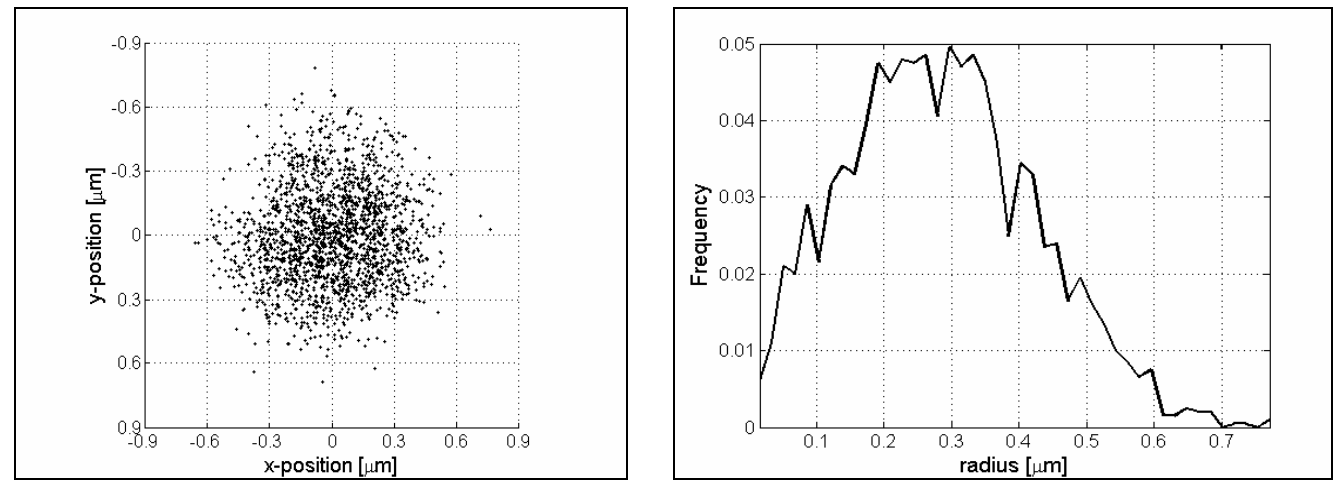

Figure 3a. $x, y$-scatter plot and histogram of a moving, tethered bead.
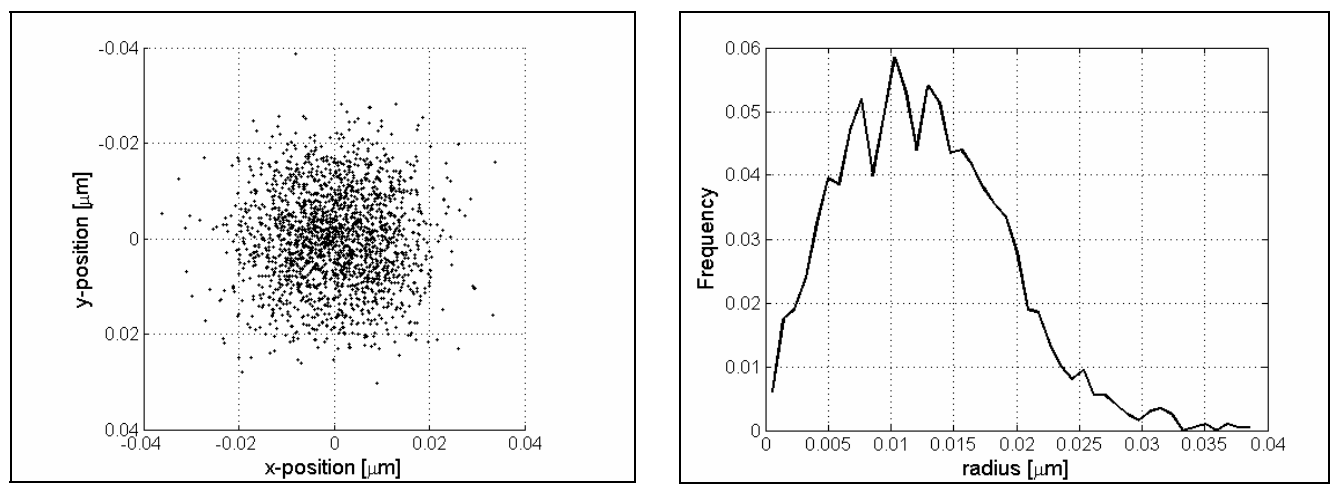

Figure 3b. $x, y$-scatter plot and histogram of a stationary bead.

The velocity of a freely moving particle can be described by a Maxwell-Boltzmann distribution if the movement is due to Brownian motion. TPM measurements are performed in three, spatial dimensions (plus time) but our image analysis is based upon two-dimensional projected data. If motion in each of the spatial dimensions is independent of the other 
two, then the Maxwell-Boltzmann distribution can be integrated over the $z$ (axial) dimension to produce the statistical description of the two-dimensional $(x, y)$ lateral case that we observe through the microscope. The resulting distribution is the Rayleigh distribution. Thus, for fitting the speed of a moving, tethered bead we use the Rayleigh expression:

$$
f(v)=A \cdot v \cdot e^{-\alpha v^{2}}
$$

where $A$ and $\alpha$ are fitting parameters and $v$ and $v^{2}$ correspond to the length and length-squared, respectively, of the velocity vector. The data and the associated fit are depicted in Figure 4 . The fit is excellent. The fitting parameter $\alpha$ can be used to estimate the "effective" mass of the DNA-bead system according to $m_{\text {eff }}=\alpha \bullet\left(2 \bullet k_{B} \bullet T\right)$ where $k_{B}$ is the Boltzmann constant and $T$ is the ambient temperature in Kelvin. The mass estimate, for the system shown in Figure 4 and taking the value of $\alpha$ found in Figure 4, is $m_{\text {eff }}=9.2 \times 10^{-10} \mathrm{~kg}$. The effective mass can also be estimated by applying kinetic theory according to

$$
\left.m=2 k_{B} T /<v^{2}\right\rangle_{x, y}
$$

where $\left\langle v^{2}\right\rangle$ is the mean-square velocity in two dimensions. The calculated mass according to equation 3 amounts to 9.0 $\mathrm{x} 10^{-10} \mathrm{~kg}$. It should not be surprising that these two values are similar.

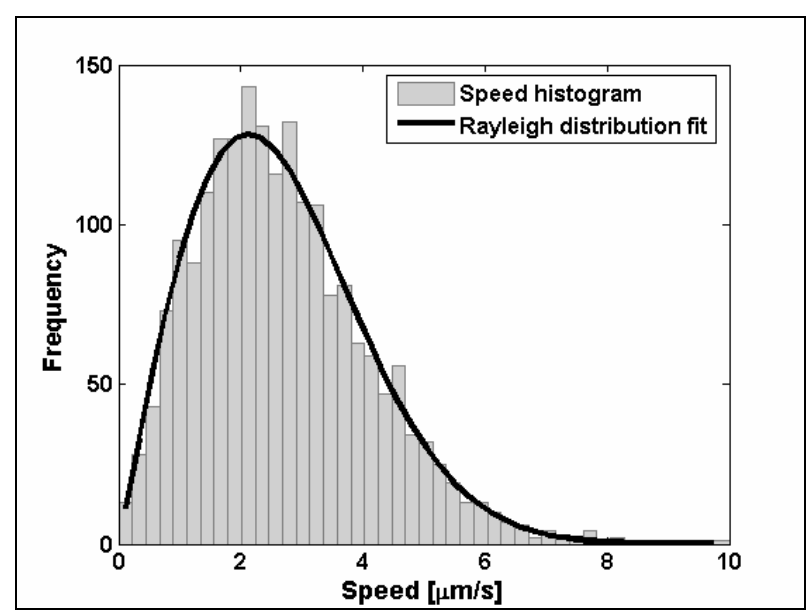

Figure 4. A Rayleigh distribution fit of a moving bead.

The mass of the $80 \mathrm{~nm}$ gold bead plus the mass of the $4861 \mathrm{bp}$ dsDNA amounts, however, to $5.17 \times 10^{-18} \mathrm{~kg}$. This value is much smaller than the effective mass calculated according to kinetic theory or by using the fitting parameter $\alpha$ from the Rayleigh distribution.

There are a number of factors that can influence the calculation of the dsDNA-bead effective mass. The experiment was performed in an aqueous environment and the surrounding hydration shell was not taken into account. Further, the dsDNA-bead combination is not freely moving but constrained by the tethering, resulting in a lower speed then one would expect in freely-moving Brownian motion. The reduced speed is reflected in a larger "mass". At this moment, it is not clear whether these two effects are sufficient to account for the larger, experimentally-calculated, effective mass.

The tethered DNA-nanobead system can be considered as behaving like a "spring/mass" harmonic oscillator according to:

$$
F=-k \cdot x
$$

where $F$ is the restoring force exerted by the system, $k$ is the spring constant and $x$ is the displacement of the system. The potential energy $U$ of the spring/mass system is given by: 


$$
U=1 / 2 \cdot k \cdot x^{2}
$$

The value of the spring constant of the tethered DNA-bead system can be derived from the Boltzmann energy distribution:

$$
P(x)=\frac{1}{Z} \cdot e^{-k \cdot x^{2} / 2 \cdot k_{B} \cdot T}
$$

where $P(x)$ is the probability of the system being in a certain energy state, $Z$ is a normalization constant; $k_{B}$ and $T$ are as before. Taking the logarithm of equation 6 gives the following formula:

$$
-\ln P(x)=\ln Z+k \cdot x^{2} / 2 \cdot k_{B} \cdot T
$$

The implications are clear. If we compute the natural logarithm of a histogram of the $x$ (or $y$ ) displacements, we should see a quadratic curve in $x$ (or $y$ ) around a "zero-point" energy. The spring constant, expressed in $\mathrm{pN} / \mu \mathrm{m}$, can then be extracted from a quadratic fit to the resulting energy profile. Figure 5 shows a typical energy profile of a $4861 \mathrm{bp}$ long dsDNA fragment tagged with a $80 \mathrm{~nm}$ large gold nanobead. The derived spring constant for the measured data shown in Figure 5 is $k=7.7 \times 10^{-2} \mathrm{pN} / \mu \mathrm{m}$. This value is in agreement with literature ${ }^{19}$.

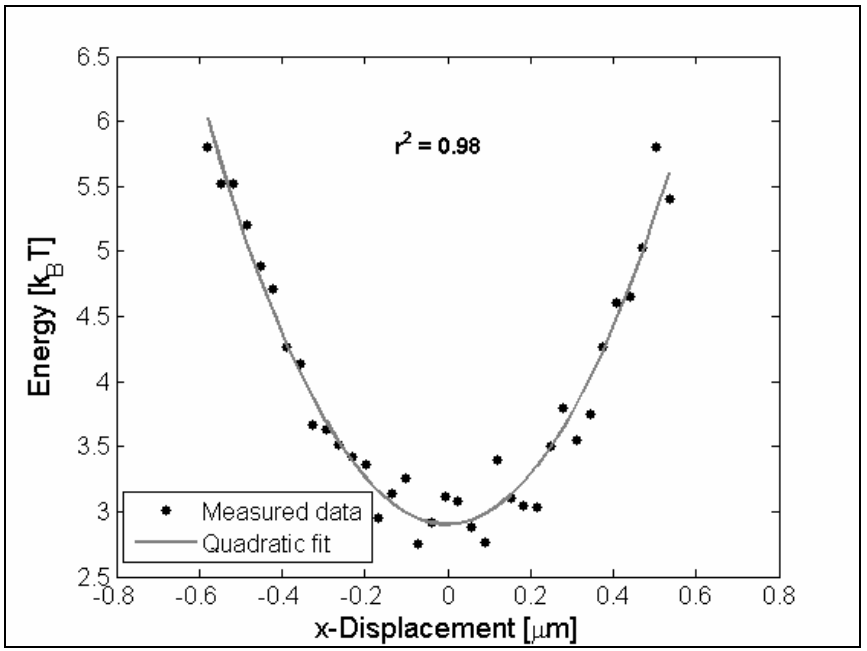

Figure 5. Energy profile of a $4861 \mathrm{bp}$ dsDNA fragment labeled with a $80 \mathrm{~nm}$ gold particle in TRIS/EDTA buffer, $\mathrm{pH}$ 8.0. The spring constant can be extracted from a quadratic fit to the experimental data.

Mono- and multivalent cations are known to compact DNA and RNA structure ${ }^{20-22}$. Various techniques have been used to study the effect of cations on the conformation of DNA and RNA. Tethered particle motion can be used to follow the change in the structure by looking at various parameters such as the rms radius and the spring constant. We have performed such experiments with the salt $\mathrm{MgCl}_{2}$ and the results are presented in Table 2 . 
Table 2. Influence of $10 \mathrm{mM} \mathrm{MgCl}_{2}$ on various parameters on a $4861 \mathrm{dsDNA}$ fragment.

\begin{tabular}{|c|c|c|}
\hline & TRIS-HCI buffer, pH 8.0 & $\mathbf{1 0 ~} \mathbf{~ M} \mathbf{M g C l}_{\mathbf{2}}$ \\
\hline$\sqrt{\left.<r^{2}\right\rangle}$ & $270 \mathrm{~nm}$ & $164 \mathrm{~nm}$ \\
\hline$\sqrt{\left.<v^{2}\right\rangle_{2 D}}$ & $3920 \mathrm{~nm} / \mathrm{s}$ & $2240 \mathrm{~nm} / \mathrm{s}$ \\
\hline Spring constant & $0.11 \mathrm{pN} / \mu \mathrm{m}$ & $0.40 \mathrm{pN} / \mu \mathrm{m}$ \\
\hline
\end{tabular}

With the addition of $10 \mathrm{mM} \mathrm{MgCl}$, the rms radius decreases by approximately $40 \%$ compared to the rms radius measured in a TRIS buffer solution. The rms velocity in two dimensions decreases by $43 \%$; and the spring constant increases by a factor of 3.6, which is not surprising considering the fact that the $(x, y)$-displacement of the nanobead decreases. The variation in these parameters can be interpreted as an indication of the compaction of the dsDNA with the addition of the salt $\mathrm{MgCl}_{2}$.

\section{CONCLUSIONS}

Using tethered-particle motion as indicated by gold nanobeads and darkfield microscopy, we have been able to observe the motion and the change in motion of bio-macromolecules when their environment is changed. We have been able to calculate various physical parameters such as the effective spring constant by applying Boltzmann statistics and the Rayleigh distribution. According to the calculated excursion number, our DNA/bead system shows a moleculedominated motion.

For a $4861 \mathrm{bp}$ long dsDNA fragment tethered onto a gold support and labeled with a $80 \mathrm{~nm}$ large gold nanobead, the addition of $10 \mathrm{mM} \mathrm{MgCl} 2$ leads to a decrease in the rms radius and the 2D-rms velocity, consequently to an increase in the spring constant. In future experiments we will be looking at interactions between specific bio-macromolecules such as dsDNA and recA.

\section{ACKNOWLEDGEMENTS}

We thank Wim van Oel and Guus Liqui Lung for their assistance in technical matters. We thank Professor Theo Odijk for many valuable discussions on the nature of DNA and Professor Simon de Vries for his assistance concerning PCR. This work was partially supported by the Bsik Research Program Cyttron.

\section{REFERENCES}

1. Weiss, S. Fluorescence spectroscopy of single biomolecules. Science 283, 1676-1683 (1999).

2. Shumaker-Parry, J.S., Campbell, C.T., Stormo, G.D., Silbaq, F.S. \& Aebersold, R.H. Probing Protein DNA Interactions Using a uniform monolayer of DNA and surface plasmon resonance. Proceedings of SPIE 3922, 158166 (2000).

3. Guille, M.J. \& Kneale, G.G. Methods for the analysis of DNA-protein interactions. Mol Biotechnol 8, 35-52 (1997).

4. Berezovski, M. \& Krylov, S., N. Using DNA-binding proteins as an analytical tool. J Am Chem Soc 125, 1345113454 (2003).

5. Brown, B. Review of Techniques for Single Molecule Detection inBiological Applications. NPL Report (2001).

6. Ishii, Y. \& Yanagida, T. Single Molecule Detection in Life Sciences. Single Molecules 1, 5-16 (2000).

7. Dietrich, H.R.C. Gold nanoparticles: A Novel Application of Spectral Imaging in Proteomics - Preliminary Results. Spectral Imaging: Instrumentation, Applications, and Analysis III (Proc. Conf. San Jose, CA USA, Jan. 22-27), Proc. SPIE 5694, $82-89$ (2005). 
8. Dietrich, H.R.C., Young, I.T. \& Garini, Y. Advances in the Development of a Novel Method to be used in Proteomics using Gold Nanobeads. Proceedings of SPIE 6092, 60920C (2006).

9. Capitanio, M. et al. Exploring molecular motors and switches at the single-molecule level. Microsc Res Tech 65, 194-204 (2004).

10. Vanzi, F., Broggio, C., Sacconi, L. \& Pavone, F.S. Lac repressor hinge flexibility and DNA looping: single molecule kinetics by tethered particle motion. Nucleic Acids Res 34, 3409-3420 (2006).

11.Vanzi, F., Vladimirov, S., Knudsen, C.R., Goldman, Y.E. \& Cooperman, B.S. Protein synthesis by single ribosomes. Rna 9, 1174-1179 (2003).

12. Finzi, L. \& Gelles, J. Measurement of lactose repressor-mediated loop formation and breakdown in single DNA molecules. Science 267, 378-380 (1995).

13. Qian, H. Quantitative Study of Polymer Conformation and Dynamics by Single-Particle Tracking. Biophys J 76, 1598-1605 (1999).

14. Qian, H. A mathematical analysis for the Brownian dynamics of a DNA tether. J Math Biol 41, 331-340 (2000).

15. Schafer, D.A., Gelles, J., Sheetz, M.P. \& Landick, R. Transcription by single molecules of RNA polymerase observed by light microscopy. Nature 352, 444-448 (1991).

16. Luengo Hendriks, C. \& al., e. DIPimage: a scientific image processing toolbox for MATLAB. Delft University of Technology.

17. Ram, S., Ward, E.S. \& Ober, R.J. Beyond Rayleigh's criterion: a resolution measure with application to singlemolecule microscopy. Proc Natl Acad Sci U S A 103, 4457-4462 (2006).

18. Segall, D.E., Nelson, P.C. \& Phillips, R. Volume-exclusion effects in tethered-particle experiments: bead size matters. Phys Rev Lett 96, 088306 (2006).

19. Blumberg, S., Gajraj, A., Pennington, M.W. \& Meiners, J.C. Three-dimensional characterization of tethered microspheres by total internal reflection fluorescence microscopy. Biophys $J$ 89, 1272-1281 (2005).

20. Bloomfield, V.A. DNA Condensation by Multivalent Cations. Biopolymers 44, 269-282 (1997).

21. Rouzina, I. \& Bloomfield, V.A. DNA Bending by Small, Mobile Multivalent Cations. Biophys J 74, 3152-3164 (1998).

22. Lambert, M.N. et al. $\mathrm{Mg}^{2+}$-Induced Compaction of Single RNA Molecules Monitored by Tethered Particle Microscopy. Biophys J 90, 3672-3685 (2006). 